\title{
APLICAÇÃO DO MODELO SWAT NA AVALIAÇÃO DO CONSUMO DE ÁGUA EM ÁREAS DE FLORESTAS PLANTADAS NA BACIA DO RIO PARÁ, ALTO SÃO FRANCISCO, EM MINAS GERAIS
}

\section{Application of SWAT Model in the evaluation of water consumption in planted forest areas in Pará river basin, Upper São Francisco, in Minas Gerais}

Evandro Luís Rodrigues

Universidade Federal de Minas Gerais, Belo Horizonte, Minas Gerais, Brasil evandromaster@gmail.com

Marcos Antônio Timbó Elmiro Universidade Federal de Minas Gerais, Belo Horizonte, Minas Gerais, Brasil timboelmiro@gmail.com

Claudia Maria Jacobi

Universidade Federal de Minas Gerais, Belo Horizonte, Minas Gerais, Brasil jacobiclau@yahoo.com

Wanderson Lopes Lamounier Instituto Federal de Educação, Ciência e Tecnologia do Sul de Minas Gerais, Passos, Minas Gerais, Brasil wandersonbioego@yahoo.com.br

Artigo recebido em 30/04/2014 e aceito para publicação em 14/11/2015

RESUMO: As florestas são de grande importância para o equilíbrio do ciclo hidrológico. Existe, porém, uma discussão acerca de que plantações florestais aumentam exageradamente o consumo de água das bacias hidrográficas, especialmente, se as plantações substituem vegetação original de porte menor como é o caso do cerrado. Este estudo teve por objetivo investigar a influência dos reflorestamentos na manutenção e no equilíbrio do ciclo hidrológico na bacia do Rio Pará, utilizando o modelo hidrológico SWAT. Os resultados evidenciam a eficiência da modelagem ambiental como método a fim detectar impactos no balanço hídrico decorrentes de alterações da cobertura vegetal. O diagnóstico revelou uma redução no consumo de $-4,26 \%$ no cenário de mata nativa, $-9,68 \%$ no cenário de reflorestamento médio e $-11,59 \%$ no reflorestamento intenso. A curva de permanência sugere a existência de grandes armazenamentos naturais indicando um alto grau de resiliência do comportamento hidrológico. Além dos efeitos da cobertura vegetal, os resultados também indicam uma estreita influência exercida pelas variáveis físicas e climáticas na governança sobre a produção hídrica da bacia. Os resultados gerados pelo modelo SWAT foram 
satisfatórios, obtendo-se estimativas confiáveis e significativas para as análises. O modelo considerou adequadamente as variáveis na sua estrutura, mostrando eficácia na representação do comportamento da bacia de maneira coerente, conforme a distribuição espacial e temporal. Os resultados apontam que as alterações a curto e médio prazo no uso e cobertura do solo de uma bacia podem acarretar impactos maiores ou menores em função das variáveis físicas e climáticas do regime hídrico.

Palavras-Chaves: florestas plantadas; vazão; evapotranspiração.

ABSTRACT: Forests are very important for the balance of the hydrological cycle. There is, however, a discussion on whether plantations increase exaggeratedly water consumption in watersheds, especially if such plantations replace original smaller-size vegetation such as in the cerrado. This study aimed to investigate the influence of reforestation in maintenance and balancing the hydrological cycle in the basin of the Pará River, Upper San Francisco, Minas Gerais, using the hydrological model SWAT. The results show the efficiency of environmental modeling as a method to detect impacts on water balance due to changes in vegetation cover. The diagnosis revealed a reduction of $4.26 \%$ in the scenario of bushland use, $9.68 \%$ on average reforestation scenario, and $11.59 \%$ in the intensive reforestation. The retention curve suggests the existence of large natural storage indicating a high degree of resilience of the hydrological behavior. Besides plant cover, the results also indicate a close influence of physical and climatic variables in governing water production in the basin. The results generated by the SWAT model were satisfactory, producing reliable and meaningful estimates for the analyses. It is noteworthy that the model adequately considered the variables in its structure, showing its effectiveness in consistently representing the behavior of the basin according to the spatial and temporal distribution. Thus, the results indicate that changes in the short and medium term in the use and land cover in a watershed can cause either higher or lower impacts depending on the physical and climatic variables of water regime.

Keywords: planted forests; flow; evapotranspiration.

\section{INTRODUÇÃO}

As florestas têm um papel fundamental na manutenção e no equilíbrio do ciclo hidrológico (GREGORY et al.,1992). Na natureza, elas exercem funções significativas no controle da produção deágua com a permanência de vazões mínimas, que garantema estabilidade e o equilíbrio dos ecossistemas aquáticos e terrestres (TUCCI e CLARKE, 1997). O uso do solo e a cobertura vegetal têm papel preponderante, pois a água, em quantidade e qualidade, é "produzida e armazenada" na área territorial da bacia hidrográfica, alimentando nascentes e tributários da calha principal (LLERENA, 2007).

Entretanto, de acordo com Lima (2012), há uma percepção de que plantações florestais levam a um consumo de água exagerado pairando muitas dúvidas quanto aos reais impactos dessas florestas na produção de água das bacias hidrográficas, especialmente, se a vegetação anterior for de porte menor como é o caso do cerrado (LIMA, 2011). De acordo com a ABRAF (2013), as áreas de plantações florestais no país correspondem a aproximadamente 5,98 milhões de hectares, sendo 3,75 milhões com eucaliptos, 1,8 milhões com pinus e 425,2 mil ha com outras espécies. Estas áreas de florestas plantadas ocupam $0,65 \%$ do território nacional e $1 \%$ do solo agropecuário.

Neste contexto, a modelagem surge como alternativa para a obtenção de informações sobre a dinâmica do comportamento, antecipação de eventos e previsão de possíveis impactos ambientais em uma bacia hidrográfica que sejam decorrentes de eventuais mudanças no uso da terra. Os modelos hidrológicos constituem instrumentos importantes para representar os sistemas, seus processos e para auxiliar na compreensão dos fenômenos que envolvem as variáveis do ciclo da água (TUCCI, 1998).

Diante desses aspectos, os modelos vêm recebendo cada vez mais destaque, configurando-se como instrumentos técnicos na gestão da água. Entre 
eles destaca-se o SWAT (Soil and Water Assessment Tool), cujas variáveis dependem do tempo ou espaço e permitem uma avaliação continuada dos fenômenos hidrológicos envolvidos. O SWAT foi desenvolvido para simular o ciclo hidrológico, produção de sedimentos e aporte de nutrientes baseado nas práticas de manejo que são efetivas na bacia hidrográfica (WU \& XU, 2006). O modelo é capaz de projetar as tendências e os efeitos sob mudanças climáticas e alterações no uso do solo. A capacidade de prever efeitos em longos períodos como resposta às transformações ambientais de causas naturais ou antrópicas que afetam os recursos hídricos em uma bacia é um aspecto importante do modelo (NEITSCH et al., 2011).

Para reproduzir as condições reais da bacia, o modelo SWAT necessita de informações sobre as características físicas e climáticas. Com esse conjunto de variáveis, o modelo faz a compartimentação da bacia baseado no Modelo Digital do Terreno (MDT) que representa as condições topográficas de declividade e as formas do relevo, associadas às informações pedológicas e de uso e cobertura vegetal. As sub-bacias são divididas em Unidades de Resposta Hidrológica (HRU), mantendo os parâmetros espacialmente distribuídos com características homogêneas que representam a heterogeneidade dos fatores que influenciam o comportamento hidrológico.

As HRU reúnem combinações únicas de uso do solo, cobertura vegetal, classes de solo e declividade, o que possibilita ao modelo refletir diferenças localizadas na vazão, escoamento superficial, percolação, fluxo lateral, fluxo subsuperficial, fluxo de retorno do aquífero raso e evapotranspiração, dentre outras condições hidrológicas para diferentes usos e tipos de solos.

Segundo Arnold et al. (1998), essas características do modelo garantem um diferencial no estudo da variabilidade do comportamento físico em diferentes partes do sistema que refletem os fluxos de energia e as interações que governam os fenômenos hidrológicos.
Neste contexto, o modelo SWAT foi utilizado para analisar a dinâmica da vazão na produção de água da bacia do Rio Pará, afetada por florestamentos em larga escala, mediante alterações no uso e cobertura vegetal visando avaliar as respectivas demandas por recursos hídricos.

\section{MATERIAIS E MÉTODOS}

\section{Área de estudo}

A bacia hidrográfica do vale do Rio Pará está localizada na cabeceira do alto Rio São Francisco no estado de Minas Gerais e suaárea corresponde a 12.300 $\mathrm{km}^{2}$, abrangendo 35 municípios. Geograficamente a bacia está situada dentro do retângulo envolvido pelas coordenadas $\mathrm{E}=451.525,57 \mathrm{~m}$ e $\mathrm{N}=7.873 .768,77 \mathrm{~m}$ e $\mathrm{E}=581.888,17 \mathrm{~m}$ e N=7.697.371,28m, Sistema UTM, Fuso 23, Hemisfério Sul, Sistema Geodésico SAD 69 (South American Datum 1969), com altitude média aproximada de $800 \mathrm{~m}$ (Figura 1). 
Figura 1 - Localização da área de estudo: Bacia do Vale do Rio Pará, MG

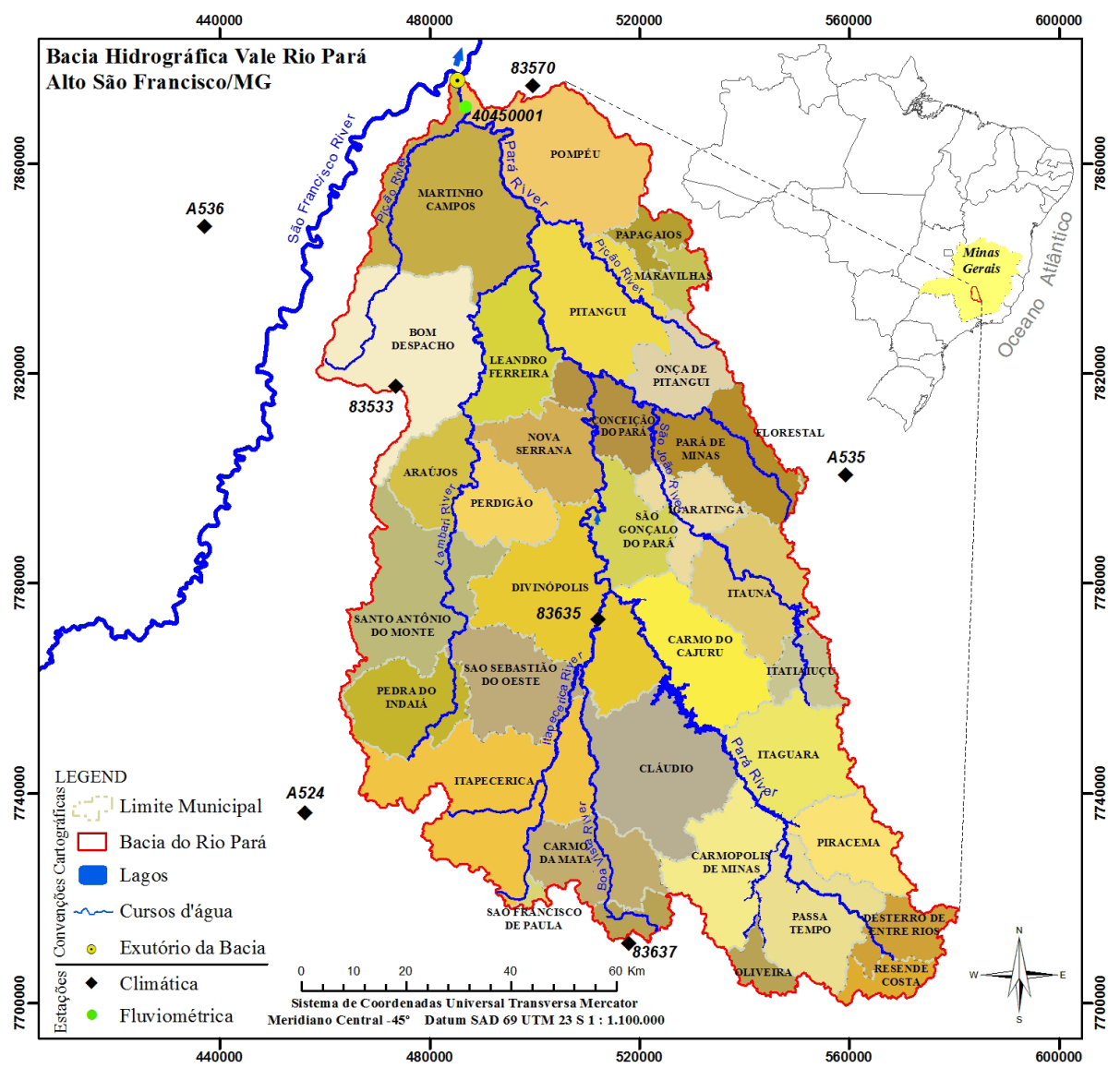

Fonte: Organização dos autores

O vale do Rio Pará tem clima quente e semiúmido conforme Köppen (1936), classificado em Aw, Cwa e Cwb, caracterizado em geral por invernos secos e verões chuvosos, com temperatura média no inverno de $16,5^{\circ} \mathrm{C}$, no verão, de $29^{\circ} \mathrm{C}$, e nas outras estações de $23,8^{\circ} \mathrm{C}$. O mês mais seco tem precipitação inferior à décima parte da precipitação do mês mais chuvoso com índice pluviométrico entre $1.200 \mathrm{~mm}$ a $1.700 \mathrm{~mm}$ anuais. O Rio Pará, afluente pela margem direita do Rio São Francisco, localizase no setor centro oeste de Minas Gerais. Suas nascentes encontram-se próximas ao município de Resende Costa, MG, adjacentes ao divisor norte da cabeceira do Rio Brumado. O talvegue apresenta extensão total de cerca de $210 \mathrm{~km}$, desenvolvendose desde a altitude $600 \mathrm{~m}$ até $1.340 \mathrm{~m}$. A vegetação que compõe a cobertura na bacia é do bioma cerrado, caracterizada pela existência de um estrato arbustivo com árvores espaçadas, retorcidas, em geral dotadas de cascas grossas e suberosas e de raízes profundas e pela existência de um estrato herbáceo-gramíneo. Entretanto, o cerrado encontra-se, em grande parte, degradado pela atividade pastoril, que é praticada de forma extensiva. A degradação é também aliada à ocupação urbana, mediante a ampliação das áreas urbanas e parcelamento do solo e do aumento dos reflorestamentos.

\section{Materiais}

Foi construído um banco de dados contendo informações sobre a topografia, uso e cobertura vegetal, pedologia e hidrografia. Informações hidroclimáticas relativas à fluviometria, precipitação, temperatura, vento, umidade e radiação solar também integraram os dados que foram utilizados 
na alimentação do modelo para fins de calibração e de representação do comportamento hidrológico da bacia.

A base de dados meteorológicos foi cedida pelo Instituto Nacional de Meteorologia - INMET a partir das estações A536, A535, A524, 83570, 83533, 83635,83637 , localizadas na área de influência da bacia. As estações forneceram os dados com a série histórica climática, constituída pelas variáveis: radiação solar $\left(\mathrm{Kj} / \mathrm{m}^{2}\right)$; precipitação pluviométrica $(\mathrm{mm})$; temperaturas máxima, média e mínima $\left({ }^{\circ} \mathrm{C}\right)$; umidade relativa do ar (\%) e velocidade do vento (m/s), no período de 1980 a 2012, com resolução temporal diária. Para calibração e validação do modelo, os dados fluviométricos foram obtidos da estação 40450001 (Porto Pará) administrada pela
Agência Nacional de Águas (figura 1).

O algoritmo usado na modelagem hidrológica foi o ArcSwat 2012 acoplado à plataforma de geoprocessamento ArcInfo 10.1 .

\section{Métodos}

O modelo hidrológico SWAT foi aplicado no ambiente da bacia hidrográfica do Rio Pará para a simulação das alterações do uso do solo sob diferentes cenários de cobertura considerando as variáveis físicas e climáticas. As etapas do trabalho e a alimentação do modelo são ilustradas na Figura 2.

Figura 2 - Fluxograma das etapas para ajuste do modelo hidrológico SWAT

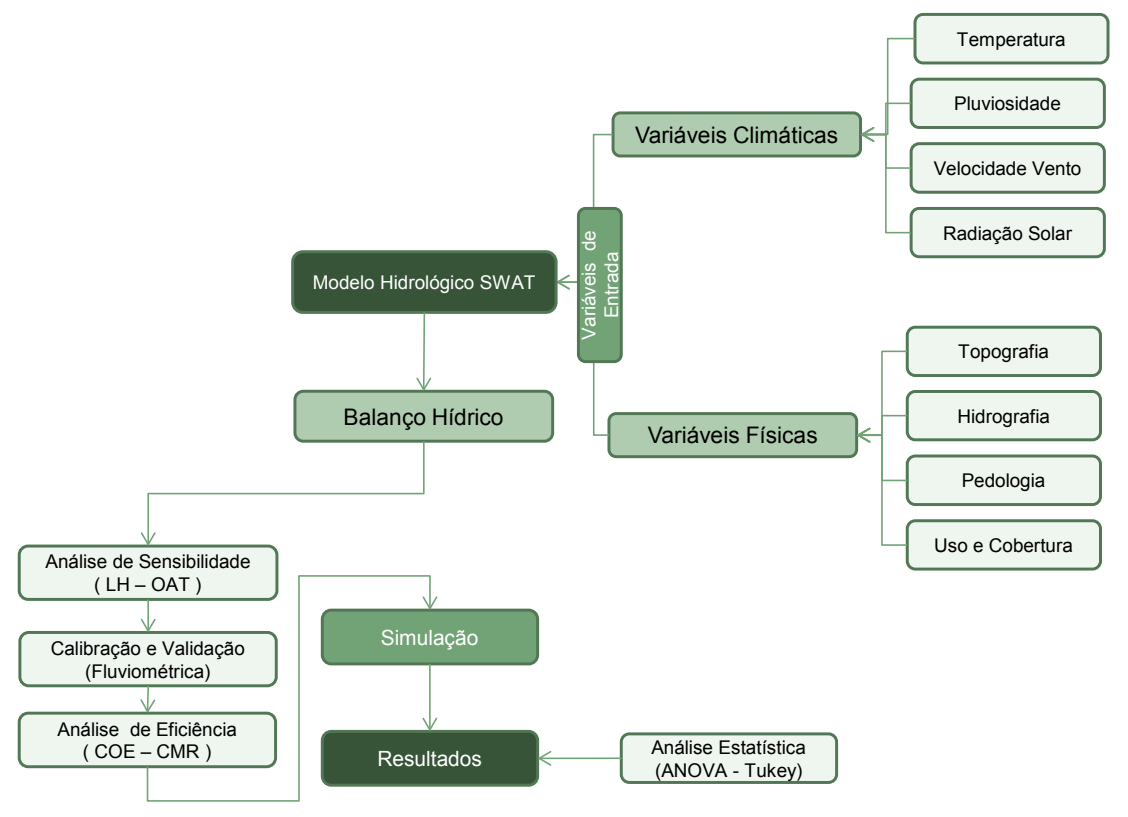

Fonte: Organização dos autores

A fase inicial consiste na avaliação da sensibilidade dos parâmetros combinando-se os métodos Latin Hypercube - LH e One-factor-At-aTime - OAT (VAN GRIENSVEN et al., 2006). O método permite identificar, classificar e restringir o número de parâmetros a serem otimizados que têm impacto significativo sobre as saídas do modelo (GREEN e VAN GRIENSVEN, 2008; SALTELLI et al., 2000).
O Latin Hypercube é baseado na simulação de Monte-Carlo, através de um método de amostragem estratificada (MCKAY, 1988; HUANG, 2015). Este método permite uma estimativa eficiente das estatísticas de saída eliminando a necessidade de inúmeras simulações (SALTELLI et al., 2000; HOLVOET, 2005). Na amostragem estratificada um conjunto $\left(\mathrm{x} 1, \mathrm{x} 2, \ldots, \mathrm{x}_{\mathrm{N}}\right)$ de dimensão $n$ é gerado a partir de uma distribuição acumulada dos valores 
iniciais. Essa amostragem é também designada como pseudoaleatória uma vez que os números aleatórios são gerados por meio de um processo determinístico.

Nesse processo, a amplitude de cada parâmetro é dividida em $n$ faixas com uma probabilidade de ocorrência igual a $1 / n$, e então o modelo faz uma combinação randômica dos parâmetros, sendo que cada faixa é testada uma única vez (VAN GRIENSVEN et al. 2006,). A amostra estratificada tem como propósito cobrir todo o espaço de amostragem dos valores iniciais dos parâmetros. Assim, uma grande vantagem do método de Monte Carlo é a combinação lógica da calibração, análise de sensibilidade e de incerteza dentro de uma única estrutura (WAINWRIGHT et al., 2014; VANROLLEGHEM et al., 2015). Para cada $n$ combinações aleatórias uma retro alimentação é passada ao modelo OAT (NARULA, 2013).

$\mathrm{O}$ método One-factor-At-a-Time (Morris, 1991), promove a alteração de apenas um parâmetro a cada simulação, gerando um efeito parcial no processo de ajuste $\mathrm{Si}, \mathrm{j}$, calculado pra cada parâmetro pela Equação 1.

$S_{i, j}=\left[\frac{\operatorname{SSE}\left(\Phi_{1}, \ldots, \Phi_{i} *(1+f), \ldots, \Phi_{p}\right)-\operatorname{SSE}\left(\Phi_{1}, \ldots, \Phi_{i}, \ldots, \Phi_{p}\right)}{f}\right]$

Onde: $\mathrm{Si}, \mathrm{j}$ é o efeito parcial do parâmetro $\Phi_{i}$ em LH no ponto $j, f$ em que é a fração obtida do parâmetro $\Phi_{i}$ modificado pela constante predefinida, e $S S E$ é a soma do erro dos quadrados.

$\mathrm{Na}$ equação 1 o parâmetro $\Phi_{i}$ é randomicamente alterado pela fração $f$, considerando $p$ parâmetros. A retro alimentação envolve a realização de $p+1$ a cada rodada onde obtém-se um efeito parcial para cada parâmetro. À medida que um parâmetro é modificado a magnitude irá depender do valor dos parâmetros restantes, esse ensaio é repetido para todas as amostras $n$. O efeito final será então calculado como a média de um conjunto dos efeitos parciais $n$ (Huang 2015). A técnica permite que as mudanças sejam atribuídas unicamente ao parâmetro alterado limitando o número de parâmetros (AHL et al., 2008, LI et al., 2009).

Como resultado, LH-OAT é um método robusto e eficiente: para $n$ intervalos por meio da técnica LH um total de $n x(p+1)$ simulações são necessárias. O LH-OAT proporciona classificações de sensibilidade com base nos efeitos finais. Assim, a sensibilidade do modelo é avaliada em toda a faixa viável através de um número de diferentes valores para outros parâmetros do modelo, incorporando assim uma quantidade limitada de interações (HOLVOET, 2005).

Portanto, a combinação desses métodos permite a identificação dos parâmetros mais sensíveis e facilita a etapa de calibração. Essa análise visa à obtenção de um ajuste adequado, pois permite a otimização dos parâmetros conferindo ao modelo uma aproximação realística às condições observadas (GREEN e VAN GRIENSVEN, 2008).

Subsequente à etapa de análise de sensibilidade foi realizada a calibração e a validação do modelo. Na calibração os valores dos parâmetros são alterados, dentro de um intervalo permitido, para que o modelo replique condições antecipadamente conhecidas do processo natural modelado (VIESSMAN e LEWIS, 2003). Nessa fase foi utilizada uma série temporal de dados aferidos em dois períodos, um para calibração (de Janeiro de 1980 a Dezembro de 1996) e outro para verificação do modelo (Janeiro de 1997 a Dezembro de 2012). No período de calibração os parâmetros de entrada do modelo são variados até que seja obtido um ajuste razoável. Na validação, os parâmetros obtidos no período de calibração são empregados para executar o modelo e o ajuste é analisado por métodos estatísticos (NEITSCH et al., 2011; ARNOLD et al., 2000).

A avaliação de desempenho do ajuste do modelo foi verificada através do coeficiente de eficiência de Nash-Sutcliffe (COE), que é o método mais utilizado para avaliação da eficiência de modelos hidrológicos, expresso pela Equação 1.

$$
C O E=1-\frac{\sum_{t=1}^{n}\left(E_{o b}-E_{c a l}\right)^{2}}{\sum_{t}^{n}\left(E_{o b}-E_{m}\right)^{2}}
$$

Onde: $E_{o b}$ é o valor observado, $E_{c a l}$ é o valor calculado, e $E_{m}$ é a média da série de valores observados no período. O COE varia de infinito negativo até o valor máximo de 1 , que representa a eficiência máxima. Além do COE foi utilizado 
também o coeficiente de massa residual (CMR), que indica quando o modelo superestima (valores negativos) ou subestima (valores positivos) os valores simulados. O CMR é expresso pela Equação 2.

$$
C M R=\frac{\sum_{i=1}^{n} E_{o b s}-\sum_{i=1}^{n} E_{c a l}}{\sum_{i=1}^{n} E_{o b s}}
$$

Onde: $E_{o b s}$ é o valor observado, $E_{c a l}$ é o valor calculado.

As simulações do ciclo hidrológico modeladas pelo SWAT são destacadas pela fase terrestre que controla a quantidade de água para o curso d'água em cada sub-bacia; e também pela fase de propagação dessas variáveis através da rede de drenagem da bacia hidrográfica para a saída. A equação no modelo SWAT que determina a produção de água superficial na bacia hidrográfica é baseada na equação do balanço hídrico expressa na Equação 3.

$$
S W_{t}=S W_{0}+\sum_{i=1}^{t}\left(R_{i}-Q_{i}-E T_{i}-P_{i}-Q R_{i}\right)
$$

Onde: SWt é a quantidade final de água no solo, t é o tempo (dias), Ri é a precipitação (mm), Qi é o escoamento superficial (mm), ETi é a evapotranspiração (mm), Pi é a percolação $(\mathrm{mm})$, e QRi é o fluxo de retorno ( $\mathrm{mm}$ ).

Para o cálculo da evapotranspiração potencial o método escolhido foi o Penman-Monteith (1965) que melhor se adaptou às condições da bacia devido à disponibilidade das informações. Ele requer séries históricas de radiação solar, temperatura do ar, umidade relativa e velocidade do vento sendo expresso pela Equação 4.

$$
\lambda E=\frac{\Delta \cdot\left(H_{n e t}-G\right)+\rho_{\text {air }} \cdot c_{p} \cdot\left[e_{z}^{0}-e_{z}\right] / r_{a}}{\Delta+\gamma \cdot\left(1+r_{c} / r_{a}\right)}
$$

onde $\lambda E$ é a densidade do fluxo de calor latente $\left(\mathrm{MJ} \mathrm{m}^{-2} \mathrm{~d}^{-1)}, E\right.$ é a taxa de evaporação profunda ( $\mathrm{mm}$ $\left.\mathrm{d}^{-1}\right), \Delta$ é a rampa da curva pressão-temperatura do vapor de saturação, $d e / d T\left(\mathrm{kPA}^{\circ} \mathrm{C}^{-1}\right), H_{n e t}$ é a radiação líquida $\left(\mathrm{MJ} \mathrm{m}^{-2} \mathrm{~d}^{-1}\right), G$ é a densidade do fluxo de calor à superfície $\left(\mathrm{MJ} \mathrm{m}^{-2} \mathrm{~d}^{-1}\right), \rho_{a i r}$ é a densidade do ar $(\mathrm{kg}$ $\left.\mathrm{m}^{-3}\right), c p$ é o calor específico à pressão constante (MJ $\left.\mathrm{kg}-1{ }^{\circ} \mathrm{C}^{-1}\right), e z^{0}$ é a pressão do vapor de saturação do ar à altura $\mathrm{z}(\mathrm{kPa}), e z$ é a pressão do vapor de água do ar à altura $z(\mathrm{kPa}), \gamma$ é a constante psicrométrica $(\mathrm{kPA}$ $\left.{ }^{\circ} \mathrm{C}^{-1}\right), r_{\mathrm{c}}$ é a resistência do dossel vegetativo $\left(\mathrm{s} \mathrm{m}^{-1}\right)$, e $r_{\mathrm{a}}$ é a resistência de difusão da camada de $\operatorname{ar}\left(\mathrm{s} \mathrm{m}^{-1}\right)$.

Como esse método incorpora um número maior de variáveis, ele oferece melhores resultados, por isso, foi escolhido para todas as simulações. Neste trabalho, foi observado o uso de dados obtidos em intervalos horários, tendo em vista que médias diárias podem não refletir a realidade das distribuições diurnas de velocidade do vento, umidade e radiação. Nestas condições, o cálculo da evapotranspiração potencial através da equação de Penman-Monteith produz melhores estimativas.

Visando avaliar os efeitos e o impacto das florestas plantadas na produção de água da bacia, foram propostos quatro diferentes cenários estabelecidos da seguinte forma:

Cenário I: denominado cenário de mata nativa, considera que o uso na bacia é correspondente a fitofisionomias típicas do bioma cerrado: campo, campo rupestre, campo cerrado, cerrado, floresta estacional semidecidual, veredas, urbanização, água; sendo mantida uma faixa de mata ciliar de 30,50 e $100 \mathrm{~m}$ em toda a extensão dos cursos d'água conforme sua largura e de $50 \mathrm{~m}$ ao redor das nascentes, de acordo com o Código Florestal. Corresponde ao cenário original sem interferência antrópica.

$\square \quad$ Cenário II: denominado cenário de uso e ocupação atual.

$\square \quad$ Cenário III: denominado cenário reflorestamento médio, considera que a bacia foi $30 \%$ reflorestada por Eucalyptus spp e Pinus spp.

Cenário IV: denominado cenário reflorestamento intenso, considera que a bacia foi $50 \%$ reflorestada por Eucalyptus spp e Pinus spp.

\section{RESULTADOS E DISCUSSÃO}

Realizado o processo de calibração para os dados de fluviometria, foi então avaliado o desempenho do ajuste do modelo SWAT e analisada sua eficiência por meio do Coeficiente de Massa Residual (CMR) e do Coeficiente de Nash-Sutcliffe 
(COE). A diferença entre os valores diário e mensal do CMR mostrou-se insignificante (entre $-0,057$ a $-0,059)$, correspondendo a um alto grau de correlação entre a vazão observada e a simulada. O resultado obtido pelo COE também foi satisfatório (entre 0,757 a 0,793), pois segundo Krysanova et al., (1998), um bom ajuste do modelo no processo de calibração ocorre quando valores das observações diárias e mensais estão no intervalo de 0,7 a 0,8 . Assim, o modelo realizou a previsão do comportamento da vazão adequadamente, tanto para os valores de pico como para os valores mínimos de escoamento de base, respondendo proporcionalmente às taxas de precipitação reais observadas na bacia (Figura 3). Estes resultados mostram que o modelo foi corretamente alinhado, interpretando as variáveis e, consequentemente, representando o comportamento hidrológico da bacia.

Figura 3 - Hidrograma da vazão diária: simulada e observada do período de calibração e validação

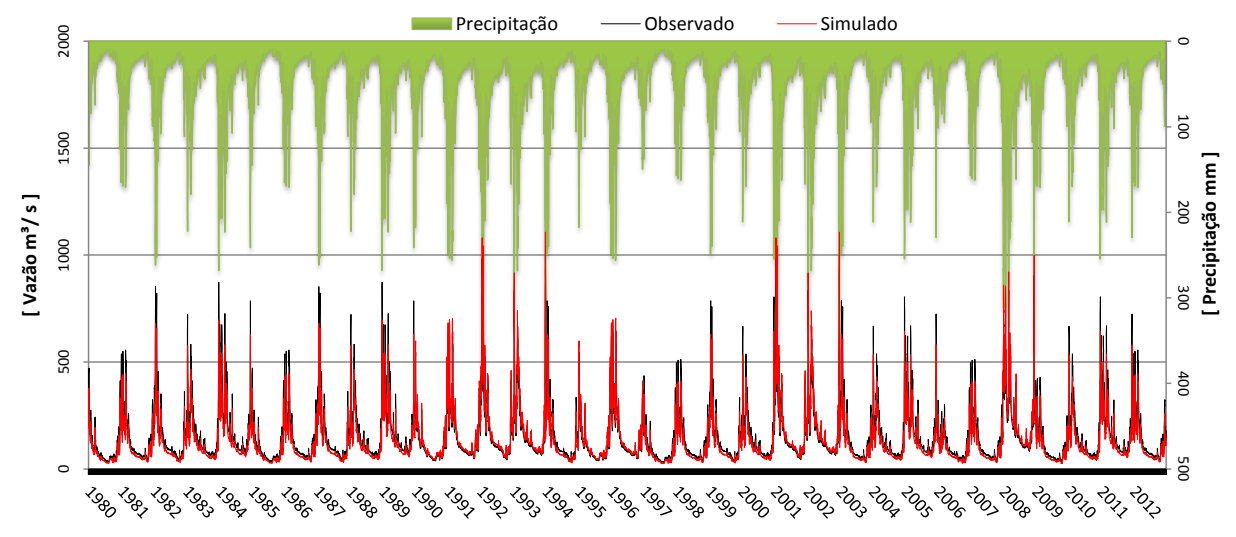

Fonte: Organização dos autores

A série simulada foi realizada para o período de 2013 a 2027 a fim de avaliar os efeitos dos diferentes cenários propostos sobre o comportamento da vazão. Os resultados indicam uma estreita influência exercida pela cobertura vegetal, marcadamente pelos usos da terra predominantes na bacia. O cenário de mata nativa e os cenários de reflorestamento médio e intenso foram comparados com o cenário de uso atual visando confrontar e avaliar as taxas de demanda por água. Os resultados referentes a essas comparações indicam que as variações na taxa de demanda hídrica no período de simulação sofreram influência quanto ao estádio de desenvolvimento destes cenários. Eles mostram que os cenários de reflorestamento apresentaram os maiores valores de déficit hídrico, variando de acordo com a idade da vegetação.

O consumo de água foi maior na fase de crescimento dos reflorestamentos, indicando que o estádio de desenvolvimento exerce influência sobre o comportamento do deflúvio. Esse fenômeno pode ser explicado pelo metabolismo vegetal mais vigoroso nesta etapa. Assim, espécies de rápido desenvolvimento, como os eucaliptos e pinus, tendem a consumir mais água do que espécies de crescimento mais lento como é o caso das fisionomias típicas do cerrado (LIMA 1993; ZHANG et al., 1999). Neste contexto, as variações percebidas na vazão podem ser creditadas à maior evapotranspiração na fase de crescimento e à posterior queda com o amadurecimento entre o décimo segundo e o décimo quinto ano do estádio de desenvolvimento. Issoreforça a tendência natural dos reflorestamentos em reduzir a evapotranspiração em idades mais longevas, o que explicaria o progressivo aumento de vazão de bacias com este tipo de vegetação após o décimo quarto ano de idade (KUCZERA, 1987; JAYASURIYA et al., 1993; VERTESSY et al., 1995; VERTESSY et al., 2001; ROBERTS et al., 2001) (Tabela 1). 
Tabela 1 - Variações na taxa de demanda hídrica em relação ao cenário de uso e ocupação atual no período de simulação quanto ao estádio de desenvolvimento (anos)

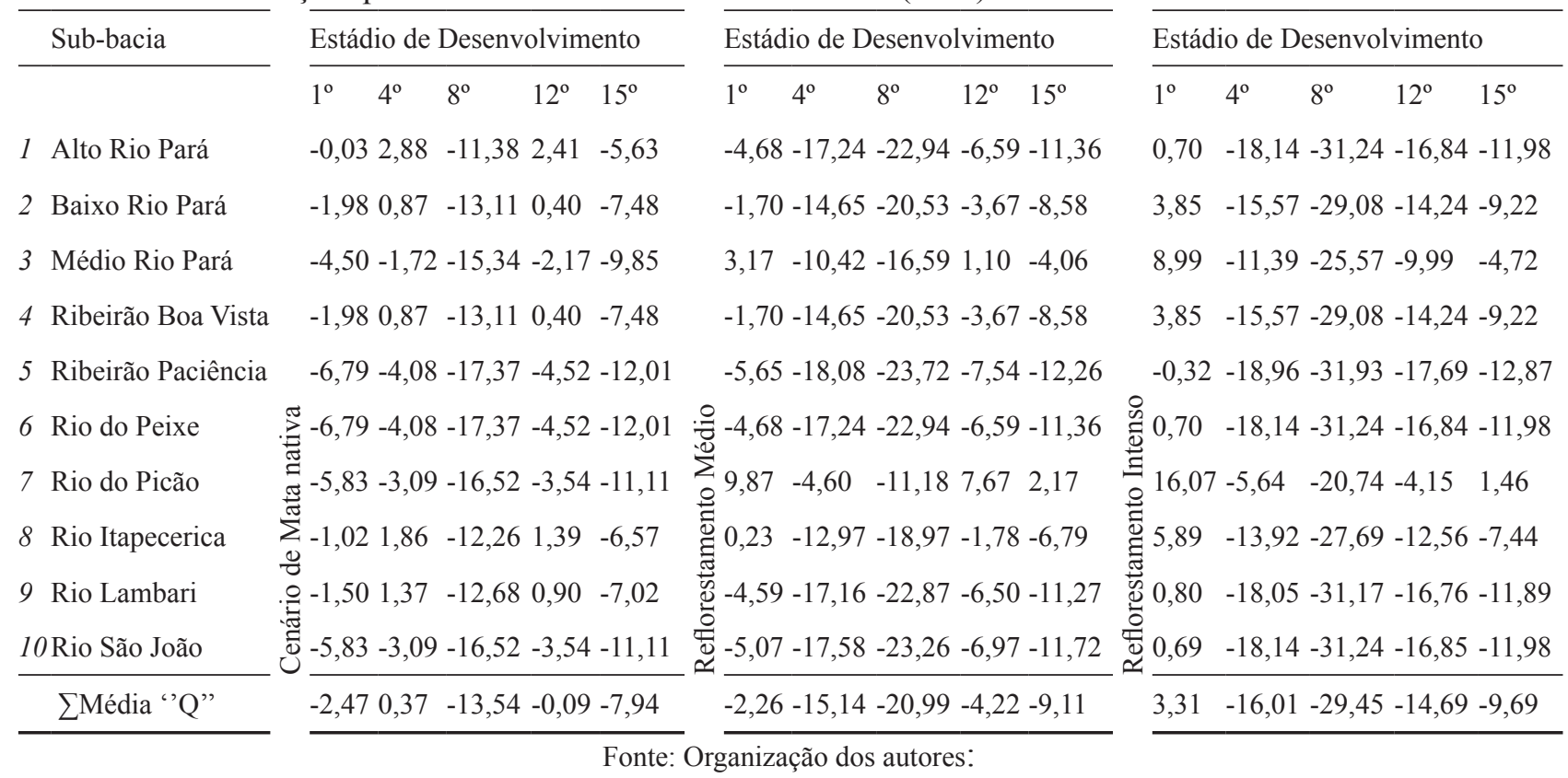

A análise do contexto indica que os efeitos hidrológicos sobre a produção de água (vazão) na bacia do Rio Pará são decorrentes, além da cobertura vegetal, de diversos componentes que atuam em nível local, tais como: relevo, pedologia, geologia e as variações do clima. Os resultados do cenário I, apesar de apresentarem uma taxa de redução da vazão da bacia ( $\sum$ Total 'Q'") em -4,26\%, evidenciam a importância da vegetação nativa aliada às áreas de preservação permanente, assim como de elementos da paisagem que contribuem para o equilíbrio do ciclo da água e para sua funcionalidade na constância de vazões mínimas maiores. Os cenários III e IV sofreram as maiores taxas de redução sobre a produção de água (-9,68 e -11,59), resultando num montante adicional de água correspondente a $24,32 \mathrm{~m}^{3} / \mathrm{s}$ e $28,15 \mathrm{~m}^{3} / \mathrm{s}$ respectivamente em cada cenário (Tabela 2 ).

Tabela 2 - Taxas de variação obtidas através da comparação entre o cenário atual e os demais cenários simulados conforme a média anual dos valores mensais da vazão $\left(\mathrm{m}^{3} / \mathrm{s}\right)$ para as sub-bacias do Rio Pará.

\begin{tabular}{|c|c|c|c|c|c|c|c|c|c|c|}
\hline & Sub-bacia & $\begin{array}{l}\text { Cenário } \\
\text { II } \\
\text { Uso } \\
\text { Atual }\end{array}$ & $\begin{array}{l}\text { Cenário I } \\
\text { Mata } \\
\text { Nativa }\end{array}$ & $\begin{array}{l}\text { Variação } \\
(\%)\end{array}$ & $\begin{array}{l}\text { Cenário } \\
\text { II } \\
\text { Uso } \\
\text { Atual }\end{array}$ & $\begin{array}{l}\text { Cenário } \\
\text { III } \\
\text { Refl. } \\
\text { Médio }\end{array}$ & $\begin{array}{l}\text { Variação } \\
(\%)\end{array}$ & $\begin{array}{l}\text { Cenário } \\
\text { II } \\
\text { Uso Atual }\end{array}$ & $\begin{array}{l}\text { Cenário } \\
\text { IV } \\
\text { Refl. } \\
\text { Intenso }\end{array}$ & $\begin{array}{l}\text { Variação } \\
(\%)\end{array}$ \\
\hline 1 & Alto Rio Pará & 40,09 & 39,34 & $-1,86$ & 40,09 & 35,44 & $-11,58$ & 40,09 & 34,68 & $-13,49$ \\
\hline 2 & Baixo Rio Pará & 24,67 & 23,74 & $-3,78$ & 24,67 & 22,50 & $-8,81$ & 24,67 & 22,01 & $-10,78$ \\
\hline 3 & Médio Rio Pará & 16,19 & 15,18 & $-6,25$ & 16,19 & 15,49 & $-4,30$ & 16,19 & 15,16 & $-6,37$ \\
\hline 4 & Ribeirão Boa Vista & 11,14 & 10,72 & $-3,78$ & 11,14 & 10,16 & $-8,81$ & 11,14 & 9,94 & $-10,78$ \\
\hline 5 & Ribeirão Paciência & 3,94 & 3,60 & $-8,59$ & 3,94 & 3,45 & $-12,48$ & 3,94 & 3,38 & $-14,37$ \\
\hline 6 & Rio do Peixe & 5,21 & 4,78 & $-8,41$ & 5,21 & 4,61 & $-11,58$ & 5,21 & 4,51 & $-13,49$ \\
\hline 7 & Rio Picão & 8,79 & 8,13 & $-7,56$ & 8,79 & 8,26 & $-6,13$ & 8,79 & 8,08 & $-8,16$ \\
\hline 8 & Rio Itapecerica & 30,51 & 29,64 & $-2,84$ & 30,51 & 28,36 & $-7,03$ & 30,51 & 27,75 & $-9,03$ \\
\hline 9 & Rio Lambari & 38,26 & 36,99 & $-3,30$ & 38,26 & 33,86 & $-11,50$ & 38,26 & 33,13 & $-13,41$ \\
\hline 10 & Rio São João & 28,17 & 26,05 & $-7,56$ & 28,17 & 24,81 & $-11,94$ & 28,17 & 24,37 & $-13,50$ \\
\hline & LTotal 'Q"' & 206,98 & 198,16 & $-4,26$ & 206,98 & 186,94 & $-9,68$ & 206,98 & 183,00 & $-11,59$ \\
\hline
\end{tabular}

Fonte: Organização dos autores 
Os resultados reforçam que o tipo de uso e a cobertura vegetal exercem um papel importante como elementos responsáveis nas variações da produção de água. Nas sub-bacias do Rio Itapecerica, Ribeirão Boa Vista, Médio e Baixo Rio Pará, a cobertura vegetal teve influência de intensidade moderada. Esse grau de influência pode ser atribuído às diferenças pedológicas ao longo da bacia, especialmente nas áreas com presença de Latossolos, pois seu maior grau de desenvolvimento, em relação às demais classes de solo, contribui com a retenção de água no solo. Além disso, há diferenças nas variáveis climáticas, com clima temperado Cwb nas cabeceiras, contribuindo para acentuar o déficit nos valores de vazão nessa região da bacia, e clima tropical Aw e inverno equatorial a jusante, em área de transição morfoclimática. A análise aponta que a magnitude dos fatores é diferenciada em virtude de características locais que podem maximizar ou atenuar os efeitos hidrológicos.

As sub-bacias do Rio Picão e Rio Lambari sofreram influência de múltiplas variáveis, evidenciando as relações quanto ao uso e cobertura vegetal predominante, ao estádio de desenvolvimento da cultura, à geomorfologia e tipos de solos, além das variáveis de ordem climática. As sub-bacias do Alto Rio Pará, Rio São João, Rio do Peixe e Ribeirão Paciência foram mais sensíveis à alteração da cobertura, indicando um alto grau de correlação quanto à vegetação e ao sistema radicular dos reflorestamentos. Essas características favorecem a infiltração, maior equilíbrio na manutenção e constância da produção de água, de forma sustentável ao longo dos anos.

Os cenários de reflorestamento médio e intenso apresentaram valores médios de vazão menores do que o cenário de mata nativa, enquanto que o cenário de calibração apresentou valor maior do que o cenário de mata nativa. Em geral, as diferenças podem seratribuídas às diferentes taxas de evapotranspiração, percolação $\mathrm{e}$ interceptação devido às diferenças na área foliar. $\mathrm{O}$ cenário de mata nativa ocupado predominantemente por fitofisionomias nativas apresentou o menor decréscimo na vazão média, cerca de $-4,26 \%$, em relação ao uso atual (cenário de calibração). Essa pequena redução pode ser atribuída ao incremento da infiltração de água no solo, ocasionada pela maior deposição de serrapilheira, bem como à proteção de áreas que mantêm a conectividade funcional do sistema como topos de morro, áreas de recarga e de preservação permanente. As áreas florestais com estrato herbáceo e rasteiro fornecem uma proteção adicional contra a evaporação diminuindo a temperatura do solo e, dessa forma, concentram maior umidade no solo devido ao sombreamento e acúmulo de matéria orgânica em decomposição proveniente das árvores (REICHERT et al., 2007).

Os cenários de reflorestamento médio e intenso, com espécies de Eucalyptus sp. e Pinus sp., apresentaram maiores decréscimos nas vazões médias, em relação ao cenário atual, cerca de $-11,47 \%$ e $-13,55 \%$. Apesar dos reflorestamentos fornecerem maior proteção ao solo e maior quantidade de matéria orgânica depositada no solo, esse comportamento pode ser explicado, principalmente, pela maior taxa de evapotranspiração das espécies estudadas e pelas perdas na interceptação (LARSON et al., 1997).

Quando se analisam os valores extremos, observa-se que os valores máximos da vazão não apresentaram uma diferença muito significativa entre os cenários simulados. Entretanto, o cenário atual foi o que gerou a vazão máxima mais elevada $\left(737,66 \mathrm{~m}^{3} / \mathrm{s} \mathrm{dia}\right)$, cerca de $1,90 \%$ superior ao cenário de mata nativa. $\mathrm{O}$ cenário que apresentou o menor valor de vazão máxima foi o de reflorestamento intenso $\left(658,77 \mathrm{~m}^{3} / \mathrm{s}\right)$, cerca de $12,68 \%$ menor que o cenário de mata nativa.

Entre as vazões mínimas, o cenário de calibração e o de mata nativa apresentaram valores superiores aos de reflorestamento. O cenário de mata nativa destacouse entre os demais porque apresentou um valor mínimo cerca de $30 \%$ superior ao de reflorestamento intenso, ou seja, uma diferença considerada extremamente elevada. Esse resultado pode ser explicado pela ação da infiltração elevada e pela menor taxa de evapotranspiração, devido também ao povoamento menos denso e menor índice de área foliar que proporciona elevação nos níveis de estocagem de água no solo, o que gera escoamento subterrâneo e, portanto, interfere no processo de percolação. Este processo é responsável pela manutenção do escoamento fluvial nos períodos de estiagem (Tabela 3$)$. 
Tabela 3 - Produção de água, na Bacia do Rio Pará, nos cenários analisados (comparação dos valores para vazões e volume)

\begin{tabular}{|c|c|c|c|c|c|c|}
\hline \multirow{2}{*}{ Cenários Simulados } & \multicolumn{3}{|c|}{ Vazões $\mathrm{m}^{3} / \mathrm{s}$} & \multicolumn{3}{|c|}{ Volume $\left(\mathrm{m}^{3} /\right.$ dia $)$} \\
\hline & Máximo & Média & Mínimo & Máximo & Média & Mínimo \\
\hline $\begin{array}{l}\text { I - Cenário de Mata } \\
\text { Nativa }\end{array}$ & 723,66 & 156,89 & 60,14 & 62.524 .224 & 13.555 .296 & 5.196 .096 \\
\hline $\begin{array}{l}\text { II - Cenário de Uso } \\
\text { Atual }\end{array}$ & 737,65 & 180,21 & 55,46 & 63.732 .960 & 15.570 .144 & 4.791 .744 \\
\hline $\begin{array}{l}\text { III - Reflorestamento } \\
\text { Médio }\end{array}$ & 698,12 & 144,21 & 48,03 & 60.317 .568 & 12.459 .744 & 4.668 .192 \\
\hline $\begin{array}{l}\text { IV - Reflorestamento } \\
\text { Intenso }\end{array}$ & 658,77 & 137,14 & 42,63 & 56.917 .728 & 11.676 .096 & 4.633 .632 \\
\hline
\end{tabular}

*Vazões compreendidas pelos valores atribuídos do período simulado, sendo determinadas para cada cenário proposto Fonte: Organização dos autores:

O resultado obtido neste estudo corrobora os trabalhos de Swank e Miner (1968), Putuhena \& Cordery (2000) e Vertessy et al. (2001), que observaram maior impacto na redução do escoamento superficial onde a cobertura com espécies de cerrado foi substituída por Eucalyptus sp. Garcia et al. (2006) obtiveram resultados semelhantes na bacia do Rio Corumbataí (SP), onde verificaram uma diminuição na vazão média anual no cenário de mata nativa típica de cerrado quando comparado ao cenário atual de uso do solo ocupado por reflorestamentos.

Para melhor compreensão dos resultados quanto às taxas de redução ocasionadas nos cenários de reflorestamentos, foi construída a curva de permanência para as vazões médias diárias observadas no cenário de calibração (Figura 4). Uma curva de permanência permite conhecer os intervalos de tempo em que as vazões foram igualadas ou excedidas. A curvatura apresentada pela sua declividade constitui um indicativo das características da bacia. Assim, uma curva plana, mais achatada, sugere que grandes armazenamentos naturais estão presentes na área da bacia. Já uma curva com forte declividade aponta a ausência de armazenamentos significativos na área da bacia (GARCIA et al., 2007; CRUZ \& TUCCI, 2008 e RIANNA et al., 2011). A forma da curva de permanência das vazões da bacia do Rio Pará (Figura 4) indica uma maior capacidade de armazenamento natural, o que sugere maior capacidade de resiliência mediante as alterações do solo. Assim, os déficits hídricos apontados nos cenários de reflorestamentos podem ter sidos atenuados por esta capacidade de retenção de água na bacia. 
Figura 4. Curva de permanência da vazão observada da bacia do Rio Pará

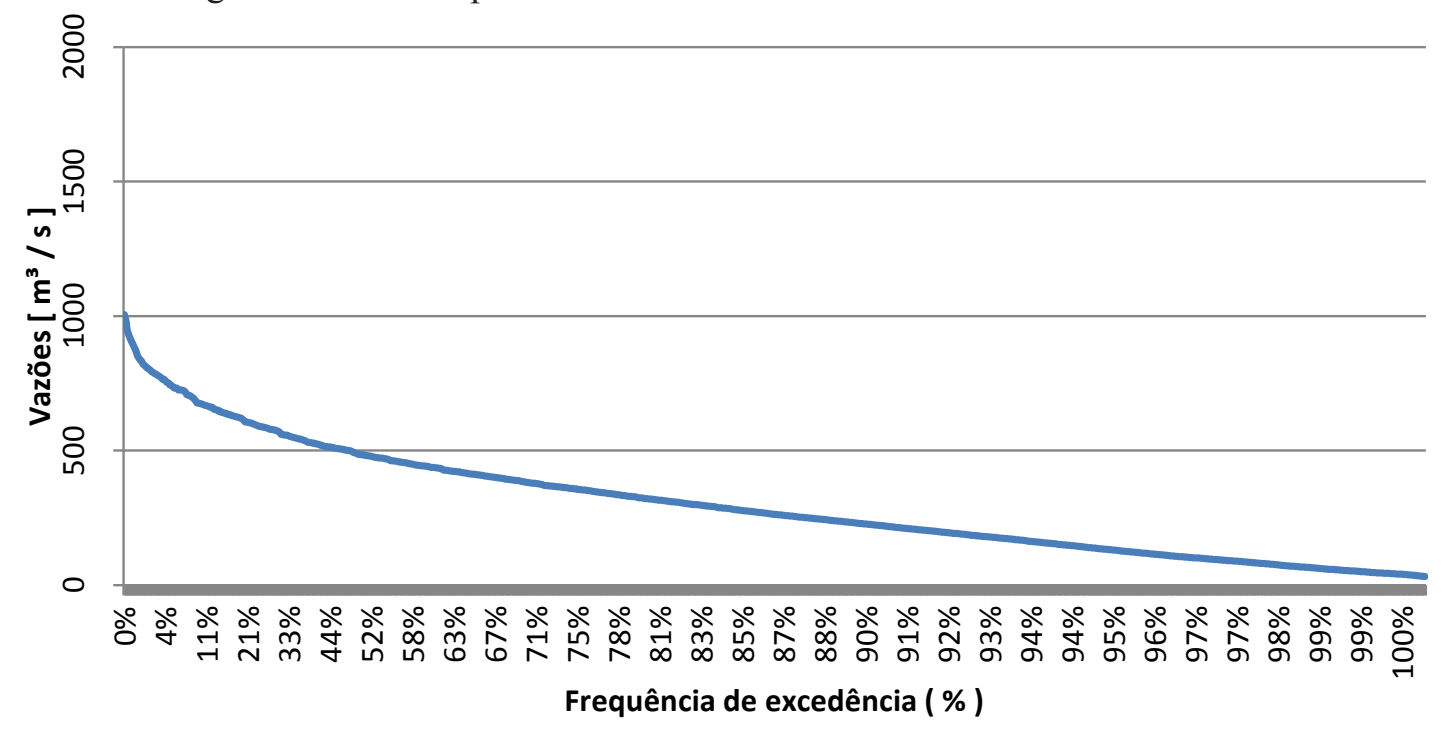

Fonte: Organização dos autores

Os resultados de vazão podem ser melhor simulados pelo SWAT, no cálculo do ciclo hidrológico avaliados quando analisados alguns dos componentes e do balanço hídrico (Tabela 4).

Tabela 4 - Variáveis do balanço hídrico do modelo SWAT para a bacia do Rio Pará

\begin{tabular}{|c|c|c|c|c|c|c|c|c|c|c|c|}
\hline Cenário & $\begin{array}{l}\text { Uso e Cobertura } \\
\text { da Terra }\end{array}$ & PREC & TRANSP & SURQ & LATQ & GWQ & PERC & SW & PET & ET & WY \\
\hline I & $\begin{array}{l}\text { Cenário de Mata } \\
\text { Nativa }\end{array}$ & 1.521 & 530 & 87 & 388 & 51 & 249 & 351 & 744 & 913 & 197,04 \\
\hline II & $\begin{array}{l}\text { Cenário de Uso } \\
\text { Atual }\end{array}$ & 1.521 & 569 & 123 & 324 & 42 & 49 & 328 & 791 & 990 & 206,98 \\
\hline III & $\begin{array}{l}\text { Reflorestamento } \\
\text { Médio }\end{array}$ & 1.521 & 617 & 120 & 437 & 48 & 187 & 184 & 812 & 990 & 182,66 \\
\hline IV & $\begin{array}{l}\text { Reflorestamento } \\
\text { Intenso }\end{array}$ & 1.521 & 784 & 104 & 560 & 26 & 258 & 88 & 989 & 1053 & 178,93 \\
\hline
\end{tabular}

PREC: precipitação (mm/dia); TRANSP: perda pelo perfil (mm/dia); SURQ: contribuição do escoamento superficial (mm/dia); LATQ: contribuição do fluxo lateral de vazão (mm/dia); GWQ: contribuição das águas subterrâneas a vazão (mm/dia); PERC: água que percola além da zona de raiz ( $\mathrm{mm} / \mathrm{dia}) ; \mathrm{SW}$ : quantidade de água no solo (mm/dia); PET: evapotranspiração potencial (mm/dia); ET: evapotranspiração real (mm/dia); WY: produção de água média $\left(\mathrm{m}^{3} / \mathrm{dia}\right)$.

Fonte: Organização dos autores

Os resultados gerados nos cenários de reflorestamento podem ser justificados pelos valores advindos da contribuição das águas subterrâneas à vazão (GWQ), daágua que percola além dazonaderaiz(PERC) e da quantidade de água no solo (SW). Esses valores ficaram muito abaixo da média dos outros cenários, ainda que tenha sido minimizado pela contribuição do fluxo lateral de vazão (LATQ). Isso justifica o resultado da variável produção de água (WY) em que em ambos os cenários de reflorestamento apresentaram valores mais baixos. Assim, o deslocamento nos resultados dos reflorestamentos em relação às outras coberturas vegetais pode ser um indicativo característico e específico dessa vegetação. 


\section{CONCLUSÕES}

Os resultados das simulações realizadas no modelo SWAT mostraram-se satisfatórios para a o presente estudo de caso na bacia do Rio Pará, permitindo as seguintes análises e conclusões:

1 - $\mathrm{O}$ modelo respondeu adequadamente às variáveis que interagem na sua estrutura, mostrando alta coerência na representação da bacia em termos de distribuição espacial e temporal.

2 - A calibração, validação e a análise de sensibilidade apresentaram comportamentos e resultados satisfatórios e compatíveis com as condições hidrológicas, climáticas e de uso e ocupação do solo atuais da bacia hidrográfica do Rio Pará.

3 - O SWAT gerou estimativas robustas e confiáveis, que foram validadas por meio das observações de séries de monitoramento, no qual os resultados apontam que as alterações em médio e curto prazo no uso e cobertura do solo de uma bacia podem acarretar impactos maiores ou menores em função das variáveis físicas e climáticas, do regime hídrico e da disponibilidade hídrica.

4 - Os resultados obtidos nos cenários de reflorestamentos apresentaram maiores taxas de consumo de água, resultando em cenários de menor produção hídrica. O cenário de mata nativa apresentou o maior valor médio diário de vazão. Entretanto, os valores máximos diários de vazão não apresentaram diferenças muito significativas entre os cenários de uso dos solos simulados. O cenário de uso atual produziu a segunda maior taxa de produção de água e os cenários de reflorestamento médio e intenso apresentaram os menores valores médios. Isso indica que as mudanças no tipo de uso, ocupação e cobertura do solo, interferem nos processos hidrológicos e, consequentemente, no regime de vazões de uma bacia hidrográfica.

5 - Mesmo que os cenários de reflorestamento contribuam para uma menor disponibilidade hídrica, pode-se inferir que os montantes de volumes de água adicionais utilizados por esse tipo de cobertura são de baixo impacto ambiental.

6 - Necessita-se de um enfoque mais sistêmico com maior ênfase às práticas de manejo que mantêm a conectividade funcional e que contribui para o equilíbrio hidrológico sustentável em sintonia com a conservação dos serviços ambientais na bacia.

\section{REFERENCIAS BIBLIOGRÁFICAS}

ABRAF. Statistical Year Book - Base Year 2012. Brasília: ABRAF, 2013. 148p.

AHL, R.S; WOODS, S.W; ZUURING, H.R. Hydrologic calibration and validation of SWAT in a snow-dominated rocky mountain watershed, Montana, U.S.A. Journal of the American Water Resources Association, Malden, v. 44, n.6, p.1411-1430, dec.2008. DOI: 10.1111/j.1752-1688.2008.00233.x

ARNOLD, J. G.; SRINIVASAN, R.; MUTTIAH, R. S.; WILLIAMS, J. R. Large area hydrologic modeling and assessment. Part I: model development. Journal of the American Water Resources Association, Temple, v. 34 , n. 1, p. 73-89, jan.1998. DOI: 10.1111/j.17521688.1998.tb05961.x

ARNOLD, J. G.; MUTTIAH, R. S.; SRINIVASAN, R.; ALLEN, P. M. Regional estimation of base flow and groundwater recharge in Upper Mississippi river basin. Journal of Hydrology, Amsterdam, v.227, n.1/4, p.21-40, jan. 2000. DOI:10.1016/S00221694(99)00139-0

Cruz,J.C.; Tucci, C.E. M. Estimativa da Disponibilidade Hídrica através da curva de permanência. Revista Brasileira de Recursos Hidricos, Porto Alegre, v.13, n.1, p.111-124, jan./mar. 2008.

GARCIA, G. J.; GAMERO, H. G.; GARCIA, L. B. R.; VETTORAZZI, C. A.; KROENERT, R.; VOLK, M.; LAUCH, A.; MEYER, B. Impacto do uso da terra na erosão do solo e no balanço e qualidade de água na bacia do Rio Corumbataí - SP. HOLOS Environment. Rio Claro, v.6, n.2, p.118-137, set. 2006.

GARCIA, A. V.; OLIVEIRA, E. C. A. de; SILVA, G. P.; COSTA, P. P. da; OLIVEIRA, L. A. de. Disponibilidade Hídrica e volume de água outorgado na micro-bacia do Ribeirão Abóbora, Município de 
Rio Verde, Estado de Goiás. Caminhos de Geografia, Uberlândia, v. 8, n.22, p.97-106, set. 2007.

GREEN, C. H.; VAN GRIENSVENA. Autocalibration in hydrologic modeling: using SWAT2005 in smallscale watersheds. Environmental Modeling and Software, Amsterdam, v.23, n.4, p.422-434, apr. 2008. DOI:10.1016/J.ENVSOFT.2007.06.002

GREGORY, S. V.; SWANSON, F. J.; McKEE, W. A.; CUMMINS, K. W. An ecosystem perspective of riparian zones. BioScience, California, v. 41, n.8, p. 540-551, sep. 1991. DOI: 10.2307/1311607

Holvoet, K.; van Griensven, A.; Seuntjens, P. ; Vanrolleghem, P.A. Sensitivity analysis for hydrology and pesticide supply towards the river in SWAT. Physics and Chemistry of the Earth, Cambridge, v. 30, n.8, p. 518-526, nov. 2005. DOI:10.1016/J. PCE.2005.07.006

Huang, J.; Xu, J.; Xia, Z.; Liu, L.; Zhang, Y.; Li, J.; Lan, G.; Qi, Y.; Kamon, M.; Sun, X.; Li, Y. Identification of influential parameters through sensitivity analysis of the TOUGH + Hydrate model using LH-OAT sampling. Marine and Petroleum Geology, Edmonton, v. 65, p. 141-156, aug. 2015. DOI:10.1016/J.MARPETGEO.2015.04.009

JAYASURIYA, M. D. A.; DUNN, G.; BENYON, R.; O'SHAUGHNESSY, P. J. Some factors affecting water yield from mountain ash (Eucalyptus regnans) dominated forests in south-east Australia. Journal of Hydrology, Amsterdam, v.150, n.2/4, p.345-367, oct. 1993. doi:10.1016/0022-1694(93)90116-Q

Köppen, W. Das geographische system der klimate. In: Köppen, W.; Geiger, R. Handbuch der klimatologie. Belin: Gerdrulier Borntraeger, 1936. 44p.

KRYSANOVA, V.; MULLER-WOHLFEIL.D.; BECKER. A. Development and Test of Spatially Distributed Hydrological Water Quality Model for Mesoscale Watersheds. Ecological Modeling, Amsterdam, v.106, n.2/3, p.261-289, mar. 1998. doi:10.1016/S0304-3800(97)00204-4
KUCZERA, G. Prediction of water yield reductions following a bushfire in ash-mixed species eucalyptus forest. Journal of Hydrology, Amsterdam, v.94, n.3/4, p.215-236, oct. 1987.

LARSON, W.E.; LINDSTROM, M. J.; SCHUMACHER, T.E. The role of severe storms in soil erosion: a problem needing consideration. Journal of Soil and Water Conservation, Ankeny, v.52, n.2,p. 90-95, mar/apr. 1997.

Li, Z; Liu W, Zhang, X., Zheng, F. Impacts of land use change and climate change variability on hydrology in an agricultural catchments on the Loess Plateau of China. Journal of Hydrology. Amsterdam, v. 377, p. 35-42, oct, 2009. DOI:10.1016/J. JHYDROL.2009.08.007

LLERENA, C.; HERMOZA; R.M.; LLERENA, L.M. Plantaciones forestales, agua y gestion de cuencas. Debate Agrario: analisis y alternativas, Lima, v.1, n.42, p.79-110, nov. 2007.

LIMA, W. P. Impacto ambiental do eucalipto. São Paulo, EDUSP. 1993. 302 p.

LIMA, W. P. Plantation forestry and water- science, dogmas, challenges. Rio de Janeiro: Instituto BioAtlântica, 2011. 64p.

LIMA,W. P.; LAPROVITERA, R.; FERRAZ, S. F. B.; RODRIGUES, C. B.; SILVA; M.M. Forest Plantations and Water Consumption: A Strategy for Hydrosolidarity. International Journal of Forestry Research, New York, v. 2012, p.1-8, nov. 2012. DOI: $10.1155 / 2012 / 908465$

McKAY, M.D. Sensitivity and uncertainty analysis using a statistical sample of input values. In: Ronen, Y. (Ed.), Uncertainty Analysis. CRC Press Inc., Boca Raton, Florida, p. 145-186, 1988.

MORRIS, M.D. Factional sampling plants for preliminary computational experiments. Technometrics, Florida, v. 33, n.2, p. 161-174, may. 1991. DOI: $10.1080 / 00401706.1991 .10484804$ 
NARULA, K. K.; GOSAIN, A.K. Modeling hydrology, groundwater recharge and non-point nitrate loadings in the Himalayan Upper Yamuna basin. Science of the Total Environment, Barcelona, v.468-469, p.S102-S116, dec. 2013. DOI:10.1016/J. SCITOTENV.2013.01.022

NEITSCH, S. L.; ARNOLD, J.G.; KINIRY, J.R.; WILLIAMS, J.R. Soil and Water Assessment ToolTheoretical Documentation. Temple: Agricultural Research Service, 2011. 647p.

PUTUHENA, W.M.; CORDERY, I. Some hydrological effects of changing forest cover from eucalypts to Pinus radiata. Agricultural and Forest Meteorology, New Haven, v.100, p. 59-72, jan.2000. DOI:10.1016/ S0168-1923(99)00086-6

REICHERT J.M., SUZUKI, L.E.A.S; REINERT, D.J. Compactação de solo em sistemas agropecuários e florestais: identificação, efeitos limites críticos e mitigação. In: CERETTA, C.A.; SILVA, L.S.; REICHERT, J.M. Tópicos em ciência do solo. Viçosa: Sociedade brasileira de ciência do solo, v. 5, p. 49134, 2007.

RIANNA, M.; RUSSO, F.; NAPOLITANO, F. Stochastic index model for intermittent regimes: from preliminary analysis to regionalization. Natural Hazards and Earth System Sciences, London, v.11, p.1189-1203, apr. 2011. DOI:10.5194/ nhess-11-1189-2011

ROBERTS, S.; VERTESSY R.A., GRAYSON R. B. Transpiration from eucalyptus sieberi (L. Johnson) forests of different age. Forest Ecology and Management, Amsterdam, v.143, n.1/3, p.153-161, apr. 2001. DOI:10.1016/S0378-1127(00)00514-4

SALTELLI, A.; TARANTOLA, S.; CAMPOLONGO, F. Sensitivity analysis as an ingredient of modelling. Statistical Science, Beachwood, v.15. n.4, p. 377-395, nov. 2000.

SWANK, W. T.; MINER, N.H. Conversion of hardwood-covered watersheds to White Pine reduces water yield. Water Resources Research, Malden, v.4, p.947-954, oct. 1968. DOI: 10.1029/ WR004i005p00947

TUCCI, C.E. M.; CLARKE, R.T. Impacto das Mudanças da Cobertura Vegetal no Escoamento: Revisão. RBRH - Revista Brasileira de Recursos Hídricos, Porto Alegre, v.2 n.1, p. 135-152, Jan./Jun. 1997.

VANROLLEGHEM, P.A.; MANNINA, G.; COSENZA, A.; NEUMANN, M.B. Global sensitivity analysis for urban water quality modelling: terminology, convergence and comparison of different methods. Journal of Hydrology, Amsterdam, p.339-352, mar. 2015. DOI:10.1016/J.JHYDROL.2014.12.056

VAN GRIENSVEN,A.; MEIXNER, T.; GRUNWALD, S.; BISHOP, T.; DILUZIO, M.; SRINIVASAN, R. A global sensitivity analysis tool for the parameters of multi-variable catchment models. Journal of Hydrology, Amsterdam, v.324, n.1/4, p.10-23, jun. 2006.

VERTESSY R.A., BENYON R.G., O'SULLIVAN S.K., GRIBBEN P.R. Relationships between stem diameter, sapwood area, leaf area and transpiration in a young mountain ash forest. Tree Physiology, Victoria, v.15, n.9, p.559-567, out. 1995.

VERTESSY, R. A.; WATSON, F. G. R; O'SULLIVAN, S. K. Factors determining relations between stand age catchment water balance in mountain ash forests. Forest Ecology and Management, Amsterdam, v.143, n.1/3, p.13-26, apr. 2001. DOI:10.1016/S03781127(00)00501-6

VIESSMAN, J. W.; LEWIS, G. L. Introduction to hydrology. New Jersey: Prentice Hall, 2002. 612 p.

WAINWRIGHT, H.M.; FINSTERLE, S.; JUNG, Y.J.; ZHOU, Q.L.; BIRKHOLZER, J.T. Making sense of global sensitivity analyses. Computers \& Geosciences, Stanford, v.65p. 84-94, apr. 2014. DOI:10.1016/J. CAGEO.2013.06.006 
WU, K. ; XU, Y.J. Evaluation of the applicability of the SWAT model for coastal watersheds in southeastern Louisiana. Journal Of The American Water Resources Association, Malden, v.42, n. 5, p.1247-1260, out. 2006. DOI: $10.1111 /$ j.1752-1688.2006.tb05298.x

ZHANG L., DAWES W.R., WALKER, G.R. Predicting the effect of vegetation changes on catchment average water balance. Australia: Cooperative Research Centre for Catchment Hydrology, 1999. 42 p. 